\title{
Usable SPACE: Security, Privacy, and Context for the Mobile User
}

\author{
Dawn Jutla \\ Department of Finance, Information Systems, and Management Science \\ Sobey School of Business \\ Saint Marys University \\ Halifax, NS, B3H 3C3 \\ Canada \\ dawn. jutla@smu.ca
}

\begin{abstract}
Users breach the security of data within many financial applications daily as human and/or business expediency to access and use information wins over corporate security policy guidelines. Recognizing that changing user context often requires different security mechanisms, we discuss end-to-end solutions combining several security and context mechanisms for relevant security control and information presentation in various mobile user situations. We illustrate key concepts using Dimitri Kanevskys (IBM Research) early 2000s patented inventions for voice security and classification.
\end{abstract}

The original version of this chapter was revised: The copyright line was incorrect. This has been corrected. The Erratum to this chapter is available at DOI: 10.1007/978-3-540-77366-5_37 